\title{
Avoidable mortality from giving tranexamic acid to bleeding trauma patients: an estimation based on WHO mortality data, a systematic literature review and data from the CRASH-2 trial
}

Katharine Ker*, Junko Kiriya, Pablo Perel, Phil Edwards, Haleema Shakur and lan Roberts

\begin{abstract}
Background: The CRASH-2 trial showed that early administration of tranexamic acid (TXA) safely reduces mortality in bleeding in trauma patients. Based on data from the CRASH-2 trial, global mortality data and a systematic literature review, we estimated the number of premature deaths that might be averted every year worldwide through the use of TXA.

Methods: We used CRASH-2 trial data to examine the effect of TXA on death due to bleeding by geographical region. We used WHO mortality data (2008) and data from a systematic review of the literature to estimate the annual number of in-hospital trauma deaths due to bleeding. We then used the relative risk estimates from the CRASH-2 trial to estimate the number of premature deaths that could be averted if all hospitalised bleeding trauma patients received TXA within one hour of injury, and within three hours of injury. Sensitivity analyses were used to explore the effect of uncertainty in the parameter estimates and the assumptions made in the model.

Results: There is no evidence that the effect of TXA on death due to bleeding varies by geographical region (heterogeneity $p=0.70$ ). Based on WHO data and our systematic literature review, we estimate that each year worldwide there are approximately 400,000 in-hospital trauma deaths due to bleeding. If patients received TXA within one hour of injury then approximately 128,000 (uncertainty range [UR] $\approx 72,000$ to 172,000 ) deaths might be averted. If patients received TXA within three hours of injury then approximately 112,000 (UR $\approx 68,000$ to $148,000)$ deaths might be averted. Country specific estimates show that the largest numbers of deaths averted would be in India and China.
\end{abstract}

Conclusions: The use of TXA in the treatment of traumatic bleeding has the potential to prevent many premature deaths every year. A large proportion of the potential health gains are in low and middle income countries.

\section{Background}

Trauma is a leading cause of death and disability. Each year, worldwide, an estimated 5.8 million people die as a result of trauma [1], many after reaching hospital. Among trauma patients who survive to reach hospital, bleeding is a common cause of death, accounting for around $40 \%$ of in-hospital trauma deaths [2].

\footnotetext{
* Correspondence: katharine.ker@lshtm.ac.uk

Clinical Trials Unit, Faculty of Epidemiology \& Population Health, London School of Hygiene \& Tropical Medicine, Keppel Street, London WC1E 7HT, UK
}

\section{Biomed Central}

(c) 2012 Ker et al; licensee BioMed Central Ltd. This is an Open Access article distributed under the terms of the Creative Commons Attribution License (http://creativecommons.org/licenses/by/2.0), which permits unrestricted use, distribution, and reproduction in any medium, provided the original work is properly cited.
The CRASH-2 trial was an international randomised controlled trial of the early administration of tranexamic acid (TXA) to bleeding trauma patients. The trial recruited 20,211 patients from 274 hospitals in 40 countries. The results show that TXA reduces mortality in trauma patients with or at risk of bleeding, with no apparent increase in side effects [3]. If given within three hours of injury, TXA reduces the risk of death due to bleeding by about a third [4]. TXA administration has been shown to be highly cost-effective in high, middle or low income countries [5]. On the basis of the 
results of the CRASH-2 trial, TXA has been included on the WHO Essential Medicines List [6].

Since publication of the trial results, TXA has been included into trauma care guidelines in many high income countries. In March 2010, the British Army incorporated TXA into combat care treatment protocols [7] and in July 2011 the UK NHS ambulance service agreed that TXA would be given to all adults and teenagers who suffer major injury in the UK. In 2011, the US Army reviewed the evidence from the CRASH-2 trial and included TXA into its trauma treatment protocols. However, bearing in mind that $90 \%$ of trauma deaths are in low and middle income countries [8], the potential of TXA to reduce premature mortality is likely to be much greater in these settings. An estimation of the number of deaths that could be averted through the use of TXA for in traumatic haemorrhage would allow better targeting of dissemination and implementation activities. In this study we used data from the CRASH-2 trial, WHO mortality database and a systematic review of the recent literature, to estimate the potential number of deaths that could be averted through the early administration of TXA to bleeding trauma patients.

\section{Methods}

\section{Estimation of effect of TXA on death due to bleeding by geographical region}

We used individual patient data from the CRASH-2 trial to assess the extent to which the effect of TXA on death due to bleeding varied according to geographical region. Hospitals participating in the CRASH-2 trial were grouped into four geographical regions: (1) Africa, (2) Asia, (3) Europe, Australia, North America, and (4) Central \& South America. Heterogeneity in treatment effect by geographical region was assessed by a $\chi^{2}$ test. We pre-specified that unless there was strong evidence against the null hypothesis of homogeneity of effects (i. e. $p<0.001$ ), the overall risk ratio (RR) would be considered to be the most reliable guide to the approximate RRs in all regions.

\section{Estimation of number of in-hospital trauma deaths due to bleeding per year}

The number of in-hospital trauma deaths that are due to bleeding and thus potentially avoidable through the early administration of TXA was estimated in three steps.

First, we obtained estimates of the number of trauma deaths $\left(\mathrm{N}_{\mathrm{T}}\right)$ by country. Since the risk of death due to bleeding may vary according to type of injury (i.e. blunt or penetrating) [9], we classified deaths as being a result of blunt trauma $\left(\mathrm{N}_{\mathrm{BT}}\right)$ or penetrating trauma $\left(\mathrm{N}_{\mathrm{PT}}\right)$. Second, we obtained data on the proportion of trauma deaths that occur in hospital $\left(\mathrm{P}_{\mathrm{H}}\right)$. The numbers of in- hospital blunt trauma deaths $\left(\mathrm{N}_{\mathrm{H}, \mathrm{BT}}\right)$ and in-hospital penetrating trauma deaths $\left(\mathrm{N}_{\mathrm{H}}, \mathrm{PT}\right)$ were then estimated according to the following equations:

$$
\begin{aligned}
& \mathrm{N}_{\mathrm{H}, \mathrm{BT}}=\mathrm{N}_{\mathrm{BT}} \times \mathrm{P}_{\mathrm{H}} \\
& \mathrm{N}_{\mathrm{H}, \mathrm{PT}}=\mathrm{N}_{\mathrm{PT}} \times \mathrm{P}_{\mathrm{H}}
\end{aligned}
$$

Third, we obtained data on proportion of in-hospital blunt trauma deaths caused by bleeding $\left(\mathrm{P}_{\mathrm{H}, \mathrm{BT}, \mathrm{BL}}\right)$ and the proportion of in-hospital penetrating trauma deaths caused by bleeding $\left(\mathrm{P}_{\mathrm{H}}, \mathrm{PT}, \mathrm{BL}\right)$. Using these estimates we derived the number of in-hospital trauma deaths caused by bleeding $\left(\mathrm{N}_{\mathrm{H}, \mathrm{T}, \mathrm{BL}}\right)$ as follows;

$$
\mathrm{N}_{\mathrm{H}, \mathrm{T}, \mathrm{BL}}=\left(\mathrm{N}_{\mathrm{H}, \mathrm{BT}} \times \mathrm{P}_{\mathrm{H}, \mathrm{BT}, \mathrm{BL}}\right)+\left(\mathrm{N}_{\mathrm{H}, \mathrm{PT}} \times \mathrm{P}_{\mathrm{H}, \mathrm{PT}, \mathrm{BL}}\right)
$$

The number of premature deaths potentially averted by TXA was then estimated by applying the relative risk reduction from the $\mathrm{CRASH}-2$ trial to the number of inhospital deaths due to bleeding as follows:

$$
\text { Premature deaths averted }=\mathrm{N}_{\mathrm{H}, \mathrm{T}, \mathrm{BL}} \times(1-\mathrm{RR})
$$

\section{Data sources}

Data from the WHO, the CRASH-2 trial and a systematic review of literature published since 2004 were used to parameterise the equations. The number of trauma deaths for each country, were obtained from the WHO for the year 2008, the most recent year for which data were available. Blunt trauma deaths were estimated by adding the number of deaths from road traffic crashes, falls and other unintentional injuries. Penetrating trauma deaths were estimated by adding the number of deaths from violence and war. Deaths from drowning, poisoning, self-inflicted injuries or burns were not included as these injuries are not usually associated with life-threatening bleeding. Estimates of the proportion of trauma deaths that are in-hospital and the proportion caused by bleeding were based on data from the CRASH-2 trial and from studies identified through a systematic review.

\section{Systematic review methods}

We searched for studies containing original data describing the epidemiology of trauma deaths. We searched MEDLINE, EMBASE and Cab Abstracts on 2 March 2011 using a combination of subject headings and key words based on the following terms; injuries, trauma, mortality, death, fatality, burden, epidemiology. We searched the internet and checked the reference lists of eligible articles. The searches were not restricted by language or publication status. To improve the applicability of the extracted data to the current patterns in trauma death epidemiology, we limited our search to studies published since 2004. 
Record screening, full text review and data extraction were performed independently by two authors (KK and $\mathrm{JK})$, with any disagreements resolved through discussion. Data were extracted on study design, setting, sample size, the proportions of deaths occurring in hospital and due to bleeding, using a pre-designed form. Studies that did not provide data on any of the parameters of interest were excluded.

To obtain a summary estimate for each parameter, the study proportions were transformed according to the Freeman Tukey variant of the arcsine square root transformed proportions to correct for over-dispersion [10]. Pooled proportions were calculated as the back-transformation of the weighted mean of the transformed proportions using the random effects model [11].

\section{Data analysis}

For each country, the number of in-hospital trauma deaths due to bleeding was calculated by applying the corresponding estimated proportions to the mortality data, as described above. For the primary analysis, the relative risk reduction from the CRASH-2 trial was applied to estimate the number of premature deaths that could be averted (1) if all patients received TXA within one hour of injury, and (2) if all patients received TXA within three hours of injury. The numbers of deaths averted in each country were combined to give an overall global estimate. To identify the countries with the greatest potential for benefit from TXA, countries were ranked in order of the estimated number of premature deaths averted.

To investigate the impact on the results of uncertainty in the parameter estimates used in the modelling, a number of sensitivity analyses were conducted. First, the analysis was repeated using the lower and upper bounds of the $95 \%$ confidence intervals for the parameter estimates to explore the effect of parameter uncertainty. Second, we repeated the analysis using the relative risk estimate for all-cause mortality rather than death due to bleeding. This analysis was conducted to take account of the possibility that some patients who do not die from bleeding because of TXA administration would nevertheless die of other causes such multi-organ failure or brain injury. Third, we repeated the analyses using the relative risk estimate for all-cause mortality with TXA when given at any time within eight hours of injury. Although, previously published subgroup analyses show that early treatment is more effective it is possible that treatment within three hours is not possible in some settings.

For each estimate, to reflect statistical uncertainty around the relative risks of TXA, an uncertainty range was estimated by calculating the numbers of deaths averted based on the $95 \%$ confidence intervals for the relative risks. The analyses were conducted using Microsoft Excel and STATA 11 (TX: StataCorp LP) software.

\section{Results}

\section{Estimation of the effect of TXA on death due to bleeding} by geographical region

Figure 1 shows the effect of TXA given within three hours of injury on death due to bleeding by geographical region. There was no evidence for heterogeneity in the effect of TXA by region $\left(\chi^{2}=1.445 ; p=0.70\right)$. The overall RRs for the effect of TXA on death due to bleeding when given within one hour $(\mathrm{RR}=0.68$; 95\% CI 0.57 to 0.82$)$ and within three hours $(\mathrm{RR}=0.72 ; 95 \% \mathrm{CI} 0.63$ to 0.83 ) of injury were therefore taken as the most reliable guide as to the approximate RRs in all regions, and were used to estimate the number of deaths that could be averted with TXA.

\section{Estimation of the annual number of in-hospital trauma deaths due to bleeding}

We identified 18 studies, described in 17 reports [12-28], which presented data on the parameters of interest and were included in the systematic review. Studies were conducted in 13 countries; USA, Canada, UK, Australia, Brazil, Denmark, Norway, Mozambique, South Africa, Italy, France, Spain and India. In addition, we obtained data collected as part of the CRASH-2 trial, which recruited patients from hospitals in 40 countries throughout the world. The study selection process is summarised in Figure 2. Data extracted from the studies are summarised in Additional File 1.

Fourteen studies [13-15,17,19-27] involving 24,831 trauma deaths provided data on the proportion of deaths occurring in-hospital; the pooled proportion was $44 \%$ (95\% CI 33 to 56\%). Five studies [3,12,16,18,28] involving 9684 deaths presented data on the proportion of blunt trauma deaths due to haemorrhage; the pooled proportion was $18 \%$ (95\% CI 13 to $23 \%$ ). Four studies $[3,12,16,28]$ involving 2256 deaths presented data on the proportion of penetrating trauma deaths due to

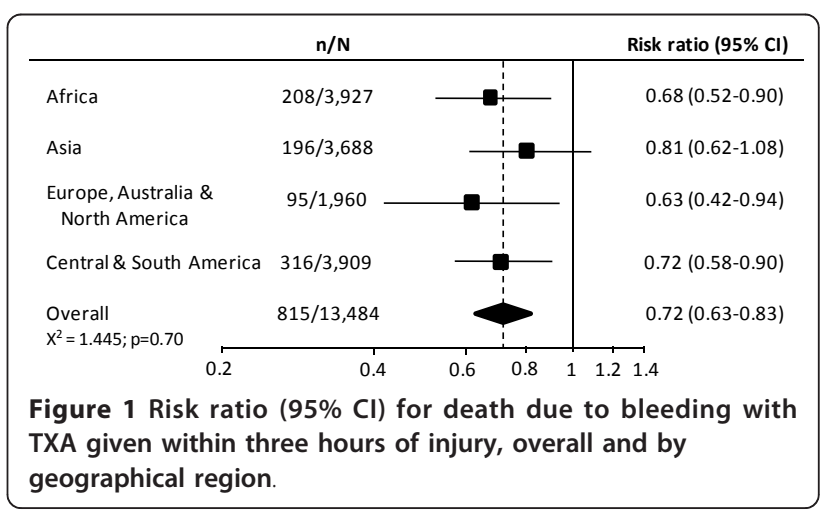




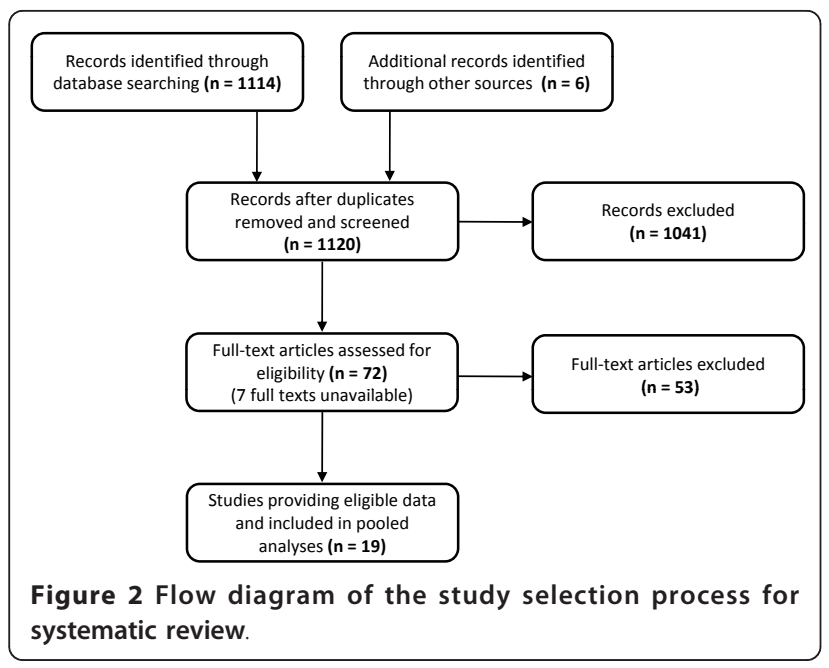

haemorrhage; the pooled proportion was 55\% (95\% CI 49 to $62 \%)$.

After applying these parameter estimates to the WHO data, we estimate that worldwide every year approximately 400,000 trauma patients die in-hospital from bleeding. If all of these patients receive TXA within one hour of injury the about 128,000 (uncertainty range $[\mathrm{UR}] \approx 72,000$ to 172,000 ) deaths could be averted. If all of these patients receive TXA within three hours of injury about 112,000 (UR $\approx 68,000$ to 148,000 ) deaths could be averted. The global distribution of number of premature deaths averted by TXA when administered within three hours of injury is shown in Figure 3.

Results for the countries where more than 1000 deaths could be averted are shown in Table 1. The largest numbers of deaths from haemorrhage and consequently the largest numbers of deaths averted are in Asia. The largest numbers of premature deaths averted are in India $(T X A \leq 1 \mathrm{hr} \approx 19,000 ; T X A \leq 3 \mathrm{hrs} \approx 16,500)$ and China $(T X A \leq 1 \mathrm{hr} \approx 17,000$; TXA $3 \mathrm{hrs} \approx 15,000)$. When ranked by the number of premature deaths potentially averted, nine of the top ten countries are low or middle income, the exception being the USA where approximately 4,000 and 3,500 deaths would be averted by TXA given within one hour and three hours of injury, respectively.

\section{Sensitivity analyses}

When the analyses were repeated using the values of the lower and upper 95\% CIs of the pooled parameter estimates, the global number of deaths averted ranged from approximately 76,000 to 198,000 if TXA is given within one hour of injury and from 67,000 to 173,000 if given with three hours of injury. When the analysis was carried out using the relative risk estimate for all-cause mortality if TXA is given within one hour $(\mathrm{RR}=0.87$; 0.78 to 0.97$)$ and within three hours $(\mathrm{RR}=0.87 ; 0.80$ to 0.94 ) of injury, the number of premature deaths averted was 52,000 (TXA $\leq 1 \mathrm{hr} \approx 12,000$ to 88,000 ; TXA $\leq 3$ $\mathrm{hrs} \approx 24,000$ to 80,000 ). When the analysis was repeated using relative risk estimate for death due to bleeding when TXA is given at any time within eight hours of injury ( $R R=0.85$; 0.76 to 0.96 ), the number of premature deaths averted was 60,000 (UR $\approx 16,000$ to 96,000 ). Finally, using the relative risk estimate for all-cause mortality when TXA is given within eight hours of injury ( $R R=0.91 ; 0.85$ to 0.97$)$, an estimated 36,000 (UR $\approx 12,000$ to 60,000 ) premature deaths could be averted.

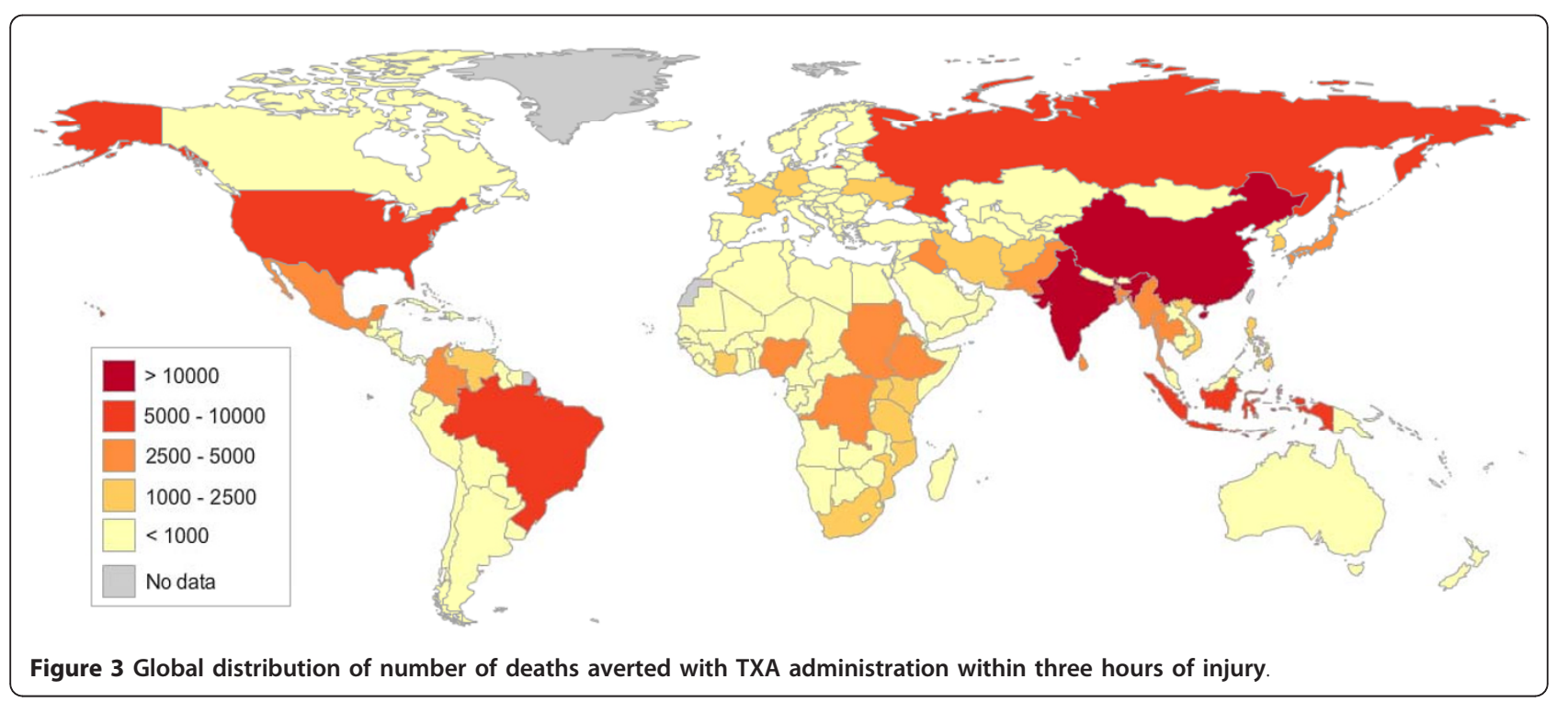


Table 1 Estimated number of premature trauma deaths averted by TXA per year

\begin{tabular}{|c|c|c|c|}
\hline & In-hospital trauma deaths from bleeding & Deaths averted TXA $\leq 1$ hour & Deaths averted TXA $\leq 3$ hours \\
\hline Worldwide & 400,467 & 128,149 & 112,131 \\
\hline \multicolumn{4}{|c|}{ Countries with $>1000$ deaths averted } \\
\hline India & 58,801 & 18,816 & 16,464 \\
\hline China & 54,241 & 17,357 & 15,187 \\
\hline Brazil & 19,187 & 6,140 & 5,372 \\
\hline Russian Federation & 16,731 & 5,354 & 4,685 \\
\hline Myanmar & 13,193 & 4,222 & 3,694 \\
\hline Iraq & 12,786 & 4,091 & 3,580 \\
\hline USA & 12,489 & 3,996 & 3,497 \\
\hline Indonesia & 11,033 & 3,531 & 3,089 \\
\hline DR Congo & 9,373 & 2,999 & 2,624 \\
\hline Sri Lanka & 8,979 & 2,873 & 2,514 \\
\hline Pakistan & 8,770 & 2,806 & 2,456 \\
\hline Ethiopia & 8,768 & 2,806 & 2,455 \\
\hline Nigeria & 8,258 & 2,643 & 2,312 \\
\hline Colombia & 7,348 & 2,352 & 2,058 \\
\hline Sudan & 7,292 & 2,334 & 2,042 \\
\hline Bangladesh & 7,210 & 2,307 & 2,019 \\
\hline Mexico & 7,059 & 2,259 & 1,976 \\
\hline Philippines & 6,119 & 1,958 & 1,713 \\
\hline Thailand & 5,572 & 1,783 & 1,560 \\
\hline Afghanistan & 4,774 & 1,528 & 1,337 \\
\hline Uganda & 4,620 & 1,478 & 1,294 \\
\hline South Africa & 4,245 & 1,359 & 1,189 \\
\hline Venezuela & 4,172 & 1,335 & 1,168 \\
\hline Kenya & 4,029 & 1,289 & 1,128 \\
\hline Tanzania & 3,969 & 1,270 & 1,111 \\
\hline Iran & 3,921 & 1,255 & 1,098 \\
\hline
\end{tabular}

\section{Discussion}

Based on WHO mortality data and a systematic review of the literature we estimate that there are about 400,000 in-hospital deaths from bleeding each year worldwide. If all hospitalised bleeding trauma patients could be treated with TXA within an hour of injury then up to 128,000 of these premature deaths could be averted. If they could be treated within three hours of injury then up to 112,000 premature deaths could averted. Although there is considerable uncertainty in the estimates even the most conservative suggest that tens of thousands of deaths could be averted every year.

We found no compelling evidence that the effect of TXA on death due to bleeding varies by geographical region. Our conclusion is based on a statistical test of interaction which is considered to be the most appropriate way to evaluate subgroup effects [29]. As recommended by methodologists, we pre-specified that unless there was strong evidence against the null hypothesis of homogeneity of effects (i.e. $p<0.001$ ), that the overall risk ratio (RR) would be considered to be the most reliable guide to the approximate RRs in all regions. We found no statistical basis to reject the null hypothesis.

The data sources used to parameterise the model are subject to a number of limitations which may have affected our results. First, although the WHO database provides the best available country-level mortality data, poor coverage and coding of mortality registration systems may affect the accuracy of the number of trauma deaths for some countries. Second, our classification of trauma deaths into blunt or penetrating trauma based on the cause of death categories in the WHO data was somewhat arbitrary and would have resulted in some misclassification. However, in the absence of accurate country-specific data, we judged that this approach would provide the most reliable estimates. Third, due to the absence of country-specific data for the proportions of deaths occurring in hospital and the proportion of deaths caused by haemorrhage, we chose to apply average global estimates. We were therefore unable to incorporate between-country variations in these parameter estimates into our analysis. Nevertheless, our estimates were derived from a systematic review of the recent 
literature and data from the CRASH-2 trial, and thus represent the most accurate estimates available. We also performed sensitivity analyses to assess the impact of uncertainty around the parameter estimates.

Since many deaths from self-inflicted injuries are not usually associated with life-threatening haemorrhage (e. g. self-poisoning, hanging) we excluded this category to avoid over-estimating the number of deaths due to bleeding. However, this is likely to have led to the exclusion of some self-inflicted deaths that were associated with haemorrhage, in which case we may have underestimated the potential of TXA administration.

Our analysis was based on a number of assumptions. We have assumed that there was no use of TXA as a treatment for traumatic bleeding prior to publication of the CRASH-2 trial results. It is possible that a small proportion of the trauma deaths in our sample did receive TXA prior to their death, which may over-estimate the number of deaths averted. However, given that any such prior use of TXA would have been minimal it is unlikely to have greatly affected our overall estimates.

The objective of our analysis was to estimate the potential number of deaths that could be averted assuming TXA use under optimal conditions, that is, when administered appropriately and within three hours of injury, to all eligible bleeding trauma patients. It is unrealistic that such conditions are consistently and fully achieved in clinical practice. For example, the opportunity to treat some eligible patients will be missed and errors in the dose used or its administration may reduce the beneficial effect of TXA.

We assumed that the results of the CRASH-2 trial could be extrapolated to all hospitalised bleeding trauma patients. The CRASH-2 trial used clinical criteria to recruit a large number of patients from 274 hospitals in 40 countries, which helps the results to be generalised widely. Whilst we acknowledge that the underlying risk of death will vary in different settings, this does not necessarily imply that that the relative effect will vary. Indeed, relative effects are often remarkably homogeneous despite differences in underlying risk. This is supported by empirical evidence from a range of trials in which the relative effects are constant across variations in baseline risk [30]. Furthermore, there is no reason to suppose that the mechanism of action of TXA would vary in different populations. However, we acknowledge that the appropriateness of such extrapolation is a matter of judgement.

A further assumption is that all trauma patients reached hospital in time to receive early treatment with TXA; that is either within one hour or within three hours of injury. Such a time frame is unlikely to be realistic in many settings where long distances and other logistical difficulties may delay arrival at hospital. For this reason we performed sensitivity analyses based on the relative effect of TXA from a more conservative estimate of time-to-treatment of within eight hours of injury, the results of which still suggest that up to 60,000 deaths could be averted. Besides, there is reason to predict that time between injury and treatment would be shorter in clinical practice than in the CRASH-2 trial as delays caused by consent procedures would be avoided [31].

In applying the RR of death due to bleeding in our primary analysis we assumed that all deaths in this group would be avoided. However, it is possible that whilst TXA may prevent death due to bleeding, some patients would die from other causes instead. If this is the case, then our primary analysis would over-estimate the number of death averted. To address this we performed a sensitivity analysis in which the effect of TXA on all-cause mortality was used. Even using this smaller relative reduction, up to 50,000 deaths could be averted.

We restricted our analysis to the potential benefit of in-hospital use of TXA. However, our parameter estimate of the proportion of in-hospital trauma deaths indicates that most trauma deaths occur before arrival at hospital. TXA is a practicable treatment suitable for use in a range of health-care settings, including pre-hospital. If TXA was used in the pre-hospital setting then many more premature deaths might be averted.

\section{Conclusions}

Our analysis shows the potential of TXA to reduce trauma deaths worldwide. Realisation of this potential is likely to require further efforts in dissemination and implementation, particularly in low and middle income settings.

\section{Additional material}

Additional file 1: Summary of data extracted from studies included in systematic review.

\section{Acknowledgements}

The CRASH-2 trial was funded by the UK NIHR Health Technology Assessment programme, Pfizer, BUPA Foundation, and JP Moulton Charitable Foundation.

\section{Authors' contributions}

KK, JK and IR designed the study. KK, JK and PP obtained the data and conducted all analyses with advice from PE and IR. KK wrote the paper with input from all other authors. All authors had full access to all the data in the study and had final responsibility for the decision to submit for publication. All authors read and approved the final manuscript.

\section{Competing interests}

The authors declare that they have no competing interests.

Received: 8 August 2011 Accepted: 1 March 2012

Published: 1 March 2012 
References

1. WHO: Injuries \& violence: the facts Geneva; 2010.

2. Sauaia A, Moore FA, Moore EE, Moser KS, Brennan R, Read RA, Pons PT: Epidemiology of trauma deaths: a reassessment. J Trauma 1995, 38(2):185-193.

3. The CRASH-2 Collaborators: Effects of tranexamic acid on death, vascular occlusive events, and blood transfusion in trauma patients with significant haemorrhage (CRASH-2): a randomised, placebo-controlled trial. Lancet 2010, 376(9734):23-32.

4. The CRASH-2 Collaborators: The importance of early treatment with tranexamic acid in bleeding trauma patients: an exploratory analysis of the CRASH-2 randomised controlled trial. Lancet 2011, 377(9771):1096-1101, 1101 e1091-1092.

5. Guerriero C, Cairns J, Perel P, Shakur H, Roberts I: Cost-effectiveness analysis of administering tranexamic acid to bleeding trauma patients using evidence from the CRASH-2 trial. PLoS One 2011, 6(5):e18987.

6. WHO: Summary of the report of the 18th meeting of the WHO Expert Committee on the Selection and Use of Essential Medicines Geneva; 2011.

7. British Forces News: Injured soldiers in Afghanistan saved by bloodclotting drug 19.01.11. 2011 [http://www.youtube.com/watch? $\mathrm{v}=0 \mathrm{j} 6 \mathrm{P} 2 \mathrm{cw} W \mathrm{RYW}$ ]

8. Peden M, McGee K, Sharma G: The injury chart book: a graphical overview of the global burden of injuries Geneva: World Health Organization; 2002.

9. Kauvar DS, Wade CE: The epidemiology and modern management of traumatic hemorrhage: US and international perspectives. Crit Care 2005, 9(Suppl 5):S1-S9.

10. Trinquart $\mathrm{L}$, Touze $\mathrm{E}$ : Pitfalls in meta-analysis of observational studies: lessons from a systematic review of the risks of stenting for intracranial atherosclerosis. Stroke 2009, 40(10):e586-e587, author reply e590.

11. DerSimonian R, Laird N: Meta-analysis in clinical trials. Control Clin Trials 1986, 7(3):177-188.

12. Boulanger $\mathrm{L}$, Joshi AV, Tortella BJ, Menzin J, Caloyeras JP, Russell MW: Excess mortality, length of stay, and costs associated with serious hemorrhage among trauma patients: findings from the National Trauma Data Bank. Am Surg 2007, 73(12):1269-1274

13. Demetriades D, Kimbrell B, Salim A, Velmahos G, Rhee P, Preston C Gruzinski G, Chan L: Trauma deaths in a mature urban trauma system: is "trimodal" distribution a valid concept? J Am Coll Surg 2005, 201(3):343-348.

14. Demetriades D, Murray J, Charalambides K, Alo K, Velmahos G, Rhee P Chan L: Trauma fatalities: time and location of hospital deaths. J Am Coll Surg 2004, 198(1):20-26.

15. Di Bartolomeo S, Sanson G, Michelutto V, Nardi G, Burba I, Francescutti C Lattuada L, Scian F: Epidemiology of major injury in the population of Friuli Venezia Giulia-Italy. Injury 2004, 35(4):391-400.

16. Dutton RP, Stansbury LG, Leone S, Kramer E, Hess JR, Scalea TM: Trauma mortality in mature trauma systems: are we doing better? An analysis of trauma mortality patterns, 1997-2008. J Trauma 2010, 69(3):620-626.

17. Evans JA, van Wessem KJ, McDougall D, Lee KA, Lyons T, Balogh ZJ: Epidemiology of traumatic deaths: comprehensive population-based assessment. World J Surg 2010, 34(1):158-163.

18. Gilroy D: Deaths from blunt trauma, after arrival at hospital: plus ca change, plus c'est la meme chose. Injury 2005, 36(1):47-50.

19. Gomez D, Berube M, Xiong W, Ahmed N, Haas B, Schuurman N, Nathens $A B$ : Identifying targets for potential interventions to reduce rural trauma deaths: a population-based analysis. J Trauma 2010, 69(3):633-639

20. Gomez JLde Segura Nieva, Boncompte MM, Sucunza AE, Louis CL, SeguiGomez M, Otano TB: Comparison of mortality due to severe multiple trauma in two comprehensive models of emergency care: Atlantic Pyrenees (France) and Navarra (Spain). J Emerg Med 2009, 37(2):189-200.

21. Masella CA, Pinho VF, Costa Passos AD, Spencer Netto FA, Rizoli S, Scarpelini S: Temporal distribution of trauma deaths: quality of trauma care in a developing country. J Trauma 2008, 65(3):653-658.

22. Meel BL: Pre-hospital and hospital traumatic deaths in the former homeland of Transkei, South Africa. J Clin Forensic Med 2004, 11(1):6-11.

23. Meisler R, Thomsen AB, Abildstrom H, Guldstad N, Borge P, Rasmussen SW, Rasmussen LS: Triage and mortality in 2875 consecutive trauma patients. Acta Anaesthesiol Scand 2010, 54(2):218-223.
24. Nizamo H, Meyrowitsch DW, Zacarias E, Konradsen F: Mortality due to injuries in Maputo City, Mozambique. Int J Inj Contr Saf Promot 2006, 13(1):1-6.

25. Potenza BM, Hoyt DB, Coimbra R, Fortlage D, Holbrook T, Hollingsworth Fridlund P: The epidemiology of serious and fatal injury in San Diego County over an 11-year period. J Trauma 2004, 56(1):68-75.

26. Singh B, Palimar $V$, Arun M, Mohanty MK: Profile of trauma related mortality at Manipal. Kathmandu Univ Med J (KUMJ) 2008, 6(23):393-297.

27. Soreide $K$, Kruger AJ, Vardal AL, Ellingsen CL, Soreide $E$, Lossius HM: Epidemiology and contemporary patterns of trauma deaths: changing place, similar pace, older face. World J Surg 2007, 31(11):2092-2103.

28. Tien HC, Spencer F, Tremblay LN, Rizoli SB, Brenneman FD: Preventable deaths from hemorrhage at a level I Canadian trauma center. J Trauma 2007, 62(1):142-146.

29. Rothwell PM: Treating individuals 2. Subgroup analysis in randomised controlled trials: importance, indications, and interpretation. Lancet 2005, 365(9454):176-186

30. Furukawa TA, Guyatt GH, Griffith LE: Can we individualize the 'number needed to treat'? An empirical study of summary effect measures in meta-analyses. Int J Epidemiol 2002, 31(1):72-76.

31. Roberts I, Prieto-Merino D, Shakur H, Chalmers I, Nicholl J: Effect of consent rituals on mortality in emergency care research. Lancet 2011, 377(9771):1071-1072.

\section{Pre-publication history}

The pre-publication history for this paper can be accessed here: http://www.biomedcentral.com/1471-227X/12/3/prepub

\section{doi:10.1186/1471-227X-12-3}

Cite this article as: Ker et al.: Avoidable mortality from giving tranexamic acid to bleeding trauma patients: an estimation based on WHO mortality data, a systematic literature review and data from the CRASH-2 trial. BMC Emergency Medicine 2012 12:3.

\section{Submit your next manuscript to BioMed Central and take full advantage of:}

- Convenient online submission

- Thorough peer review

- No space constraints or color figure charges

- Immediate publication on acceptance

- Inclusion in PubMed, CAS, Scopus and Google Scholar

- Research which is freely available for redistribution

Submit your manuscript at www.biomedcentral.com/submit
C) Biomed Central 